\title{
BMJ Open Group interventions for amyotrophic lateral sclerosis caregivers in Ireland: a randomised controlled trial protocol
}

\author{
Tom Burke, ${ }^{\oplus 1,2}$ Jennifer Wilson O'Raghallaigh, ${ }^{1}$ Sinead Maguire, ${ }^{3}$ Miriam Galvin, ${ }^{\oplus 2}$ \\ Mark Heverin, ${ }^{2}$ Orla Hardiman, ${ }^{2,3}$ Niall Pender ${ }^{1,2}$
}

To cite: Burke T, Wilson O'Raghallaigh J, Maguire S, et al. Group interventions for amyotrophic lateral sclerosis caregivers in Ireland: a randomised controlled trial protocol. BMJ Open 2019;9:e030684. doi:10.1136/ bmjopen-2019-030684

- Prepublication history and additional material for this paper are available online. To view these files, please visit the journal online (http://dx.doi. org/10.1136bmjopen-2019030684).

Received 26 March 2019 Revised 21 August 2019 Accepted 23 August 2019

A Check for updates

C Author(s) (or their employer(s)) 2019. Re-use permitted under CC BY-NC. No commercial re-use. See rights and permissions. Published by BMJ.

${ }^{1}$ Department of Psychology, Beaumont Hospital, Dublin, Ireland

${ }^{2}$ Academic Unit of Neurology, Trinity Biomedical Sciences Institute, University of Dublin Trinity College, Dublin, Ireland ${ }^{3}$ Department of Neurology, Beaumont Hospital, Dublin, Ireland

\section{Correspondence to} Dr Tom Burke, Department of Psychology, Beaumont Hospital, Dublin, Ireland; burket2@tcd.ie

\section{ABSTRACT}

Introduction Amyotrophic lateral sclerosis (ALS) is a rapid and fatal motor disease marked by progressive physical impairment due to muscle weakness and wasting. It is multidimensional with many patients presenting with cognitive and/or behavioural impairment. Caregivers of patients with ALS, commonly non-paid immediate family members, often take primary responsibility for the complex care needs of patients in non-medicalised setting, and many as a consequence experience caregiver burden, anxiety, and/or depression.

Methods and analysis This randomised controlled trial (RCT) will use randomisation to allocate $n=75$ caregivers of patients with ALS from the national ALS clinic into three groups with an equal distribution. The RCT consists of two intervention groups and a wait list control (treatment as usual [TAU]) group. The intervention arms of the trial consist of a 'mindfulness-based stress reduction' and 'building better caregivers' manualised group-based intervention, with 9 and 6 weekly sessions, respectively. The TAU group will have access to intervention at the end of the trial period. Primary outcomes are self-report questionnaires on anxiety and depression symptoms, with caregiver burden and quality of life considered secondary outcomes. Assessment will commence at baseline, immediately following the intervention period, and after a period of 12 weeks to assess the effectiveness and efficacy of participating in an intervention. Patient cognitive and behavioural data will also be considered. Means of treatment and control groups at Time 0 and 1 will be analysed using mixed model multivariate analysis of variance followed by analysis of variance, and treatment effect-sizes will be calculated. This RCT protocol is preresults and has been registered with an international database resulting in an International Standard Randomised Controlled Trials Number (ISRCTN53226941). Ethics and dissemination Ethics approval was obtained from the Beaumont Hospital Medical Research Ethics Committee. Results of the main trial will be submitted for publication in a peer-reviewed journal.

\section{INTRODUCTION}

Amyotrophic lateral sclerosis (ALS) is a progressive neurodegenerative condition characterised by rapid loss of motor function, and is associated with cognitive and behavioural impairment. People with ALS

\section{Strengths and limitations of this study}

This study will be conducted as a large randomised controlled trial concerning group-based interventions aimed to reduce psychological distress in caregivers of patients with amyotrophic lateral sclerosis (ALS).

- The secondary outcomes of this study will offer opportunity to better understand the interconnectedness of psychological distress on caregiver burden and quality of life.

- International awareness of burden and psychological distress in caregivers of patients with ALS has outpaced the available evidence on caregiver-specific treatments.

- A detailed profile of caregivers' psychological well-being and patient data allow for an intricate analysis of mediating factors

- Using a single site design is a limitation to this study.

lose functional independence, for example, being able to walk, communicate or perform basic self-care tasks as the disease progresses. Caregivers of patients with ALS, commonly non-paid immediate family members, often take primary responsibility for the complex care needs of patients outside of the medical setting. In addition to the physical symptoms, cognitive impairment occurs in up to $50 \%$ of patients with ALS, with mild-to-moderate behavioural impairment reported in up to $70 \%$ of patients with ALS, independent of cognitive status. ${ }^{1}$ Most commonly, executive dysfunction (eg, higher order functions such as planning and organisation) and apathy (demotivation) are reported..$^{2-4}$ While physical symptoms have a negative impact on caregivers ${ }^{5-7}$ so too do cognitive and behavioural symptoms. ${ }^{8-10}$ Clinically elevated levels of burden have been reported in approximately $50 \%$ of caregivers, ${ }^{11}{ }^{12}$ with neuropsychological symptoms significantly predicting burden over and above the effect of physical decline. ${ }^{13}$ There is evolving evidence that caregivers' psychological status and 
coping strategies also influence the mental and physical outcome of patients with ALS. ${ }^{14-17}$ However, the presence of cognitive/behavioural changes in patients with ALS is often under-recognised by healthcare professionals, and the burden of care associated with these changes is not currently fully addressed in clinical practice. ${ }^{18}$

Previously, we have described interventions conducted with, and designed for, patients and caregivers with ALS, as well as other populations, for example, traumatic brain injury, brain tumour, stroke, Alzheimer's disease $(\mathrm{AD})$, Parkinson's disease, Huntington's disease and multiple sclerosis. ${ }^{19}$ Some caregiver-based interventions which show a reduction in psychological distress for caregivers through psychoeducation, which is the structured presentation of information about cognitive impairment, expected caring issues, stress management and techniques to manage the patients' behaviour. ${ }^{20}$ Group-based psychoeducational programmes for family caregivers that target specific problem areas are more effective than other intervention modalities. ${ }^{21}$

Many caregivers and family members recount anecdotal evidence noting the therapeutic effect of individualised or group-based music sessions, ${ }^{22}$ and indeed counselling and support sessions have been shown to significantly delay nursing home placement for persons with $\mathrm{AD}$, improving mood and caregiver quality of life (QoL). ${ }^{23}$ Mindfulness-based stress reduction (MBSR), which can be administered in an individual or group setting, has been shown to promote a person's ability to cope with the experience and management of negative emotions, mitigating the effects of disease burden, promoting psychological adaptation. ${ }^{24}$ Considering both patient and caregivers, people who practice mindfulness have been shown to be more capable of responding to stressful situations without an automatic or otherwise maladaptive reaction, that is, avoiding coping strategies. MBSR has been shown to be a protective factor against caregiver burden in ALS. ${ }^{25}$

Developing skill-based group interventions to enhance or teach caregivers new methods of problem solving are beneficial as problem solving and reasoning skills of caregivers are an important determinant of caregiver well-being. ${ }^{26}$ In wider neurodegenerative conditions, cognitive-behavioural therapy (CBT) and peer-support/ counselling group therapy aim to improve mood and QoL and to decrease uncertainty in illness. ${ }^{27}$ Peer-support groups provide the opportunity to share personal feelings and concerns, encourage mutuality and validation and overcome feelings of social isolation. ${ }^{28}$

Studies among patients with dementia show that behavioural problems are significant predictors of exhaustion leading to caregivers' mental and physical health problem. ${ }^{29}$ Identification of cognitive decline and behavioural changes in a multidisciplinary clinic can lead to a rapid implementation of psychologically tailored interventions for behaviour change, which in turn can reduce caregiver anxiety and depression. ${ }^{30}$ Indeed, assessment of individualised QoL may be seen itself as an intervention, with the potential to influence the QoL of that individual through self-evaluation and reflection and/or their appraisal of QoL as the respondent identifies life domains of importance to them. ${ }^{31}$

Recent longitudinal findings suggest that in ALS patient-caregiver dyads caregivers' experience of burden is associated with a higher baseline of anxiety and depression, which leads to a reduced QoL as the disease progresses. ${ }^{32}$ External factors, such as additional homecare and finance do not appear to significantly impact the clinical profile of caregiver burden. ${ }^{11}$ Management of caregiver burden requires targeted intervention aimed specifically at anxiety management, increasing resilience-based strategies and reducing symptoms of low mood and anxiety. ${ }^{19}$ The presence of anxiety and low mood in caregivers at their initial clinic visit is predictive of developing caregiver burden as ALS progresses, and therefore, a time-sensitive intervention designed to increase coping and specifically reduce anxiety and/or low mood for ALS caregivers could in turn reduce caregiver burden. A recent review of caregiver burden in ALS carers details caregiver factors and their associations with psychological distress, with an itemised breakdown of the outcomes measures used, for example, anxiety, depression, which suggests that caregivers are at risk for psychological distress and group interventions may diminish burden. ${ }^{33} \mathrm{~A}$ further study ${ }^{34}$ suggests that the prevalence of anxiety and depression in ALS caregivers lies at 37\%, with $25 \%$ respectively.

While there are multiple genetic, neurobiological, psychological and social factors that precipitate, predispose and perpetuate depression and anxiety, ${ }^{35-37}$ CBT interventions have the most extensive evidence base for treating anxiety and depression during short-term intervention blocks. There is also a growing body of evidence from controlled trials, meta-analyses and systematic reviews that group-based $\mathrm{CBT}^{38}$ can be effective for mildto-moderate anxiety and depression. Specifically in relation to ALS, there is limited evidence available for the efficacy or effectiveness of psychological interventions for ALS caregivers conducted with the methodological standard of a randomised controlled trial (RCT). Others ${ }^{39}$ report that caregivers expressed a clinical need for psychological support, however there was a low demand for joint patient-caregiver CBT sessions and their study was stopped early due to low recruitment (see Caga et al, ${ }^{40}$ for a recent narrative review incorporating non-trial intervention studies with ALS caregivers).

In the proposed study, we aim to assess the effectiveness and efficacy of psychologically tailored interventions which may support a stepped-care approach in ALS caregiver group interventions. Stepped care is a service model appropriate for use in contexts where there is a demand for treatment; where treatment resources are limited; the severity of service-users' problems range from mild to moderate and there is evidence to show that low-intensity treatments may be effective for less complex and less severe clinical problems. In adult mental health services, 
there is some evidence that stepped care has advantages over usual care, particularly for anxiety and depression. $^{4142}$

\section{Study objectives}

With a goal of developing improved clinical service for ALS caregivers, we aim to determine which intervention, if any, within a 6-8 weeks framework leads to a reduction in psychological distress. In doing so, we aim:

- To evaluate the effectiveness, efficacy and tolerability of low-intensity interventions for mild-to-moderate anxiety, depression, QoL and burden in caregivers of patients using a cohort of Irish ALS caregivers;

- To inform best practice regarding the mental health needs of caregivers of patients with ALS both nationally, and internationally;

- To evaluate what ALS-specific patient factors predict caregiver outcomes regarding the efficacy and effectiveness of intervention programmes;

- To identify a standard intervention programme suitable for ALS caregivers, which in turn will define a clinic-based pathway for future enrolment to such an intervention.

The study is designed to meet a gap in the current literature and begin to build a clinically meaningful management strategy for caregivers who experience anxiety and/ or depression, with a view to having a secondary impact on caregiver burden. An objective of this study is to inform the development of appropriate future management guidelines and strategies for healthcare professionals, both nationally and internationally.

\section{METHODS AND ANALYSIS \\ Design}

This study is an RCT of group-based interventions for caregivers of people with ALS. The trial is a two-arm assessment of effectiveness and efficacy of group-based interventions (experimental treatment arm 1: CBT; experimental treatment arm 2: MBSR) with a TAU control arm. The TAU control arm is a waiting list control cohort, as currently there is no intervention provided. The trial will be conducted and reported according to ConsolidatedStandards of Reporting Trials (CONSORT) guidelines. ${ }^{43}$ Figure 1 illustrates the proposed protocol structure of the RCT. This study has been registered with an international database resulting in an International Standard Randomised Controlled Trials Number (ISRCTN53226941: https://doi.org/10.1186/ ISRCTN53226941). The ISRCTN registry is a primary clinical trial registry recognised by WHO and International Committee of Medical Journal Editors that accepts clinical research studies. This site will host interim modifications if needed.

\section{Setting and participants}

Participants will be informal caregivers (non-paid caregivers) of patients with ALS routinely attending the

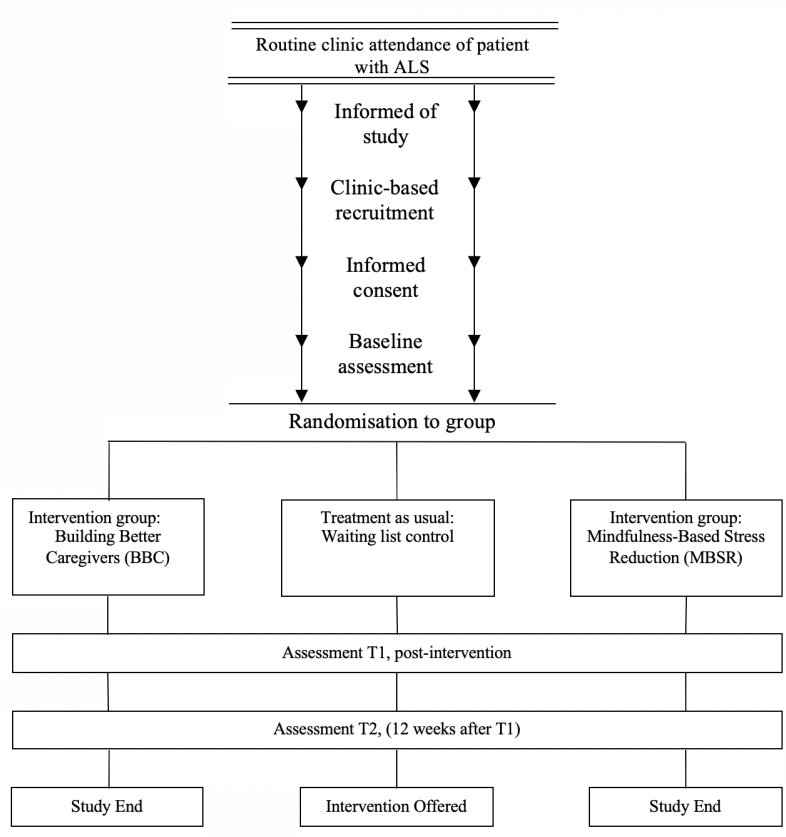

Figure 1 Flow chart of RCT study protocol. ALS: amyotrophic lateral sclerosis.

national ALS multidisciplinary clinic at Beaumont Hospital, Dublin, Ireland. Caregivers will be approached to take part in the study by a lead investigator, or an appointed research assistant from the national ALS multidisciplinary clinic, held weekly at the National Centre for Neurology, with recruitment beginning from 13June 2019 and continuing to 27 November 2020. Potential participants will be recruited within 6 months of their inaugural visit. Once consent is obtained, outlined below, they will be invited to complete measures of anxiety, and depression using validated measures employed with ALS caregivers routinely. Following screening, caregivers will be invited to attend a routine preliminary interview-based assessment. Previous engagement with psychological services, and personal and family history will be clarified during the interview to determine the severity and complexity of the case to appropriately identify and manage risk. Caregivers who present with symptoms of risk to themselves or others that is persistent and pervasive suicidal ideation and intent will be referred for high-intensity treatments. This will involve an initial review for an on-call psychiatrist within the recruitment setting, and a referral to mental health services through their general practitioner, as needed. This will be monitored throughout the study and will inform the safety procedure for participants. Participants will be randomly assigned to a treatment arm or a TAU group, which are outlined in greater detail below i.e., intervention and control arms.

\section{Inclusion and exclusion criteria}

Caregivers will be included regardless of relationship type (spouse/child/parent), although the patient must have a diagnosis of ALS over another motor neuron disease. Caregivers will be aged 18 years and over for consent 
purposes. As this intervention is interested in anxiety and low mood as a primary outcome, potential participants must endorse symptomatology of anxiety and/or depression on the primary outcome measures to be included in this study. The minimum requirement for eligibility to the study is a score of 1 on the primary outcome measure, indicating self-reported symptomatology. Consequently, participants who do not endorse anxiety and/or depression symptomatology will be excluded from this study. Furthermore, caregiver cases will be excluded if there is a comorbid active psychiatric condition present; if capacity to consent is diminished; if low levels of literacy would deem the intervention too demanding; if there are neurological or significant healthcare complications or if there is active addiction to state-altering substances. A person presenting with symptoms of persistent and pervasive suicidal ideation or intent will not be deemed eligible to take part in the study. This will be screened using the Patient Health Questionnaire-9 (PHQ-9) item relating to suicide, and an endorsement $\geq 2$, which will prompt the above-mentioned protocol in relation to risk.

\section{Patient and public involvement}

A 'patient and public involvement panel' was not specifically recruited to inform the design, recruitment, conduct or dissemination plan for this study.

\section{Randomisation}

Matching and randomisation will be used when assigning cases to groups. Matching procedures will be used to minimise baseline differences between treatment and control groups. Randomisation will be used to prevent bias in assigning cases to groups. Groups of three recruited cases will be matched as closely as possible on the following variables: age, gender, anxiety and depression profile and patient characteristics (outlined further below) in line with a priori power calculations.

Each case in a group of three will be randomised to an arm of the study with the aid of a computer pregenerated, random number sequence. The demographic and clinical characteristics of cases in treatment and control groups will be presented in a table in the report on the study. Blinding will occur at two levels. Those engaging with potential participants at the time of recruitment will be blinded to the group allocation process. Furthermore, data will be entered under coded variables, for example, groups $\mathrm{A}-\mathrm{C}$, and the statistician will be blinded to the corresponding group legend during analyses. A data audit will be facilitated and conducted by the Trinity College Dublin Academic Unit of Neurology research manager.

For transparency in reporting, the flow of participants through each stage of the study from appropriate referral, through screening, for time 0,1 and 2 assessments (pre-assessment, postassessment and follow-up assessment) will be documented in a CONSORT flow diagram for consistency. ${ }^{43}$ The CONSORT flow diagram allows for greater transparency regarding the recruitment strategy of participants into an intervention study. This will allow for identification of the number of participants who were enrolled, allocated to a treatment group, followed-up and the reasons why some may have been excluded from analysis, for example, attrition.

\section{Interventions and control arms}

The intervention arms of the study will be conducted by a principal clinical psychologist who has received the standardised formal training in the interventions, and is a master trainer in this regard. Intervention sessions last approximately $90 \mathrm{~min}$ each, and may be co-facilitated by another trainer. The interventions, as outlined below, have not been adapted from the original intervention, and a group will consist of nine participants at a time.

\section{Intervention arm 1: building better caregivers}

For treatment arm 1, we will run the building better caregivers $(\mathrm{BBC})^{44}$ programme, as developed by Stanford University. It is described in brief below. In accordance with CBT-based programmes, there is a treatment manual for facilitators to follow-this ensures the standardised delivery of the programme. There is an accompanying workbook in accordance with CBT practice that will incorporate the homework tasks, which are an essential component of CBT. This programme contains six weekly sessions. A detailed breakdown of the intervention structure and content can be seen in table 1 . In this programme, a range of strategies are used to optimise mood management and self-regulation skills through a number of core components such as skill mastery, modelling, exploration of care-partner behaviours and social support building.

\section{Intervention arm 2: mindfulness-based stress reduction}

This 8-week class is modelled on the stress reduction clinic $^{45}$ and will focuses on mindfulness meditation to support a new understanding of how stress affects one's life, and how life can be lived more fully with a mindful mentality. The sessions for the group are broadly categorised as: being awake; ways of seeing; being at home in your body; meeting stress; responding to stress; mindful communication; mindfulness in daily life; taking care of oneself—looking back/moving Forward. A breakdown of the intervention can be seen in table 2 .

\section{Control arm: treatment as usual}

This cohort will act as a non-intervention control group for the study, recruited as a waiting list cohort. On briefing, the potential participants will be informed that they may be assigned to this intervention arm. Should participants be assigned to the TAU arm of the study, they will receive first preference on a group-based intervention should the study show improvement on the primary outcome measures. Participants will be interviewed, as outlined, in order to investigate potential other sources of therapeutic intervention during this research study.

\section{Compliance}

Participants will be invited to take part in this study, and for those who consent, they will meet with a trained 
Table 1 Overview of treatment arm 1: building better caregivers

\begin{tabular}{|c|c|c|c|}
\hline Session & Topic & Structured content* & Key component† \\
\hline 1 & Introduction to workshop & $\begin{array}{l}\text { Introduction to workshop } \\
\text { Group introductions } \\
\text { Thoughtful breathing } \\
\text { Tools for improving fatigue } \\
\text { Challenging care partner behaviours } \\
\text { - Introduction to action plans } \\
\text { Closing }\end{array}$ & $\begin{array}{l}\text { - Psychoeducation } \\
\text { - Modelling } \\
\text { Behavioural intervention } \\
\text { - Action planning }\end{array}$ \\
\hline 4 & $\begin{array}{l}\text { Making Decisions and Stopping } \\
\text { Unhelpful Thinking }\end{array}$ & $\begin{array}{l}\text { Feedback } \\
\text { Care partner's challenging behaviour } \\
\text { Making decisions } \\
\text { - Getting help } \\
\text { Stopping unhelpful thinking } \\
\text { - Making an action plan } \\
\text { Closing }\end{array}$ & \\
\hline 6 & $\begin{array}{l}\text { Communication, Working } \\
\text { with Healthcare Providers and } \\
\text { Planning for the Future }\end{array}$ & $\begin{array}{l}\text { Feedback } \\
\text { Reviewing difficult care partner } \\
\text { emotions } \\
\text { Working with healthcare systems/ } \\
\text { providers } \\
\text { Communication skills } \\
\text { Looking back and planning for the } \\
\text { future } \\
\text { Closing }\end{array}$ & \\
\hline
\end{tabular}

${ }^{*}$ Referred to as activities in the manualised approach.

†Key components across all topics.

facilitator prior to the group-based intervention beginning. At this time, they will also meet a research assistant who will support their engagement throughout the study. As is routine practice with intervention-based groups, this will involve the research assistant phoning the consented participant prior to the beginning of the group, to remind them of the upcoming session, and indeed to facilitate any homework-based queries they may have. The facilitator and research assistant will then meet with the participants individually at the group-based intervention, to support their engagement at the group. A recent pilot study employing this design yielded a $96 \%$ completion rate throughout the intervention phase. ${ }^{46}$ Participant tolerability will be reported and considered in relation to intervention attendance. 
Table 2 Overview of treatment arm 2: mindfulness-based stress reduction

\begin{tabular}{|c|c|c|c|}
\hline Session & Topic & Structured content & Key component \\
\hline 1 & Being awake & $\begin{array}{l}\text { Becoming aware of thoughts, emotions and } \\
\text { sensations in the present moment. } \\
\text { Review of guidelines and rules. } \\
\text { Introduction round. }\end{array}$ & $\begin{array}{l}\text { Meditation training: } \\
\text { bodyscan }\end{array}$ \\
\hline 2 & Ways of seeing & $\begin{array}{l}\text { Becoming aware of automatic pilot. } \\
\text { Analysing vs sensing. } \\
\text { Recognising behavioural, cognitive and emotional } \\
\text { patterns that arise when working with difficulties. }\end{array}$ & $\begin{array}{l}\text { Meditation training: } \\
\text { awareness of breath } \\
\text { Seeing exercise }\end{array}$ \\
\hline 3 & At home in the body & $\begin{array}{l}\text { Becoming aware of physical sensation in movement. } \\
\text { Understanding physical, emotional and cognitive } \\
\text { reactions to pleasant events. }\end{array}$ & $\begin{array}{l}\text { Meditation training: } \\
\text { mindful movement } \\
\text { Walking meditation }\end{array}$ \\
\hline 4 & Meeting stress & $\begin{array}{l}\text { Orientation to the full stress reaction and physical, } \\
\text { emotional and cognitive sequelae. } \\
\text { Education regarding enduring and chronic stress } \\
\text { models. }\end{array}$ & $\begin{array}{l}\text { Meditation training: sitting } \\
\text { exercise }\end{array}$ \\
\hline 6 & Mindful communication & $\begin{array}{l}\text { Becoming aware of communication styles and } \\
\text { reactions, including our own expectations as listener } \\
\text { and speaker. } \\
\text { Learning to observe our reactions to the } \\
\text { communication of others. }\end{array}$ & $\begin{array}{l}\text { Meditation training: sitting } \\
\text { exercise } \\
\text { Paired work }\end{array}$ \\
\hline 7 & $\begin{array}{l}\text { A day of } \\
\text { mindfulness }\end{array}$ & Deepen meditation practice: 7-hour session. & Meditation training \\
\hline 8 & Mindfulness in daily life & $\begin{array}{l}\text { Making the connection between meditation practice } \\
\text { and daily life. } \\
\text { Becoming aware of patterns that are self-destructive } \\
\text { vs self-nourishing. }\end{array}$ & $\begin{array}{l}\text { Meditation training: } \\
\text { bodyscan, lake }\end{array}$ \\
\hline
\end{tabular}

Note: Psychoeducation and discussion are integral components of each of the modules also.

\section{Outcome measures and administration}

Questionnaires will be administered to participants as a baseline before the intervention, immediately following the intervention, and again after 3 months. The primary aim of the study is to indicate the extent to which an intervention group may lead to improvements in caregiver anxiety and depression (effectiveness), and if these improvements were maintained at 3 months follow-up (efficacy). To do that, the following measures are considered primary outcomes: PHQ-9 (total score) ${ }^{47}$; General Anxiety Disorder Questionnaire-7 (GAD-7: total score) ${ }^{48}$ and the Hospital Anxiety and Depression Scale (HADS: total score). ${ }^{49}$ Scores obtained from the Zarit Burden Interview (total score) ${ }^{50}$ and McGill Quality of Life Questionnaire (total score $)^{51}$ will be considered as secondary outcome measures. Cut-offs will be used for severity categorisation according to standardised test development and validation. The timeline of metrics to be administered is outlined in table 3 . To check that potential confounding factors are balanced between the groups, baseline questionnaires will collect data on: demographics, medication use, family composition and relevant medical history, ethnicity, education, employment status, current occupation, number of siblings and self-determined level of support network. Caregivers' own coping style may influence their experience of stress, as has been shown in caregivers of patients with ALS. ${ }^{26}$ Therefore, we will administer the Coping Inventory for Stressful Situations (CISS) at baseline and consider this a mediating factor.

There are a number of mediating factors which may also influence the primary and secondary outcome data, which we aim to consider. As ALS is a clinically heterogenous condition with distinct cognitive and behavioural subphenotypes, ${ }^{1}$ the accompanied patient at the time of recruitment will be requested to consent to their routine clinic data that relates to cognitive and behaviour classification and functional status, for example, the ALS Functional Rating Scale-Revised ${ }^{52}$ to be used. Cognitive 
Table 3 Baseline and outcome measures with assessment time-point noted

\begin{tabular}{|c|c|c|c|c|}
\hline Outcome & Instrument & TO & T1 & T2 \\
\hline \multicolumn{5}{|l|}{ Demographics } \\
\hline Caregiver and patient characteristics & Demographic questionnaire & $\bullet$ & & \\
\hline Psychological distress & HADS & $\bullet$ & - & $\bullet$ \\
\hline Anxiety & GAD-7 & - & - & $\bullet$ \\
\hline \multicolumn{5}{|l|}{ Secondary outcomes } \\
\hline Caregiver burden & ZBI & - & $\bullet$ & $\bullet$ \\
\hline Quality of life & McGill-QoL & $\bullet$ & $\bullet$ & $\bullet$ \\
\hline \multicolumn{5}{|l|}{ Mediator } \\
\hline Coping style & CISS & - & & \\
\hline Behavioural change (patient) & $\mathrm{BBI}$ & - & & $\bullet$ \\
\hline Disease progression (patient) & ALSFRS-R & $\bullet$ & & $\bullet$ \\
\hline
\end{tabular}

ALSFRS-R, Amyotrophic Lateral Sclerosis Functional Rating Scale - Revised; BBI, Beaumont Behavioural Inventory; ECAS, Edinburgh Cognitive Assessment Scale; GAD-7, General Anxiety Disorder Questionnaire;HADS, Hospital Anxiety and Depression Scale; PHQ-9, Patient Health QuestionnaireZBI, Zarit Burden Interview.

and behavioural data are routinely collected at the host institution's motor neuron disease multidisciplinary team clinic in the form of the Edinburgh Cognitive Assessment Scale (ECAS) $)^{53}$ and the Beaumont Behavioural Inventory $(\mathrm{BBI})^{54}$ at initial clinic visit and follow-up. Categorical cut-off points for impairment from both ECAS and BBI will be used. ${ }^{355}$

\section{Data storage}

All data arising from this research project will be confidential to the research team. Completed paper assessment protocols, marked with case numbers, will be held in secure filing systems. Codified numerical data from paper protocols will be stored on secured systems and in SPSS files. Participants' names will not be stored in these electronic systems, and records will be identified by case number only. A master-list linking case numbers with clients' names and contact details will be held in a password-protected computer file. Journal articles, presentations and other publications arising from the research will contain aggregated data so individual cases may not be identified.

\section{Data availability statement}

The datasets generated and/or analysed during the current study during this study will be included in the subsequent results publication, and will be uploaded alongside the manuscript as supplementary material, in line with the research ethics approval.

\section{Statistical analysis}

Determining effectiveness and efficacy

Internal consistency reliability coefficients will be computed for all scales, to determine how reliably constructs were measured in the study. Internal consistency reliability for all multi-item scales will be evaluated with Cronbach's $\alpha$. Improvement rates for treatment and control groups will be calculated and compared for the primary outcome. A comparison will be made for all cases in an intent-to-treat analysis and also for treatment completers. Treatment completers are defined as those who attend at minimum of $75 \%$ of the sessions, as outlined in the current literature. ${ }^{56}$ In intent-to-treat analyses, multiple imputation procedures will be used to impute missing data.

Means of treatment and control groups at time 0 and 1 will be analysed using mixed model multivariate analysis of variance (MANOVA) followed by analysis of variance (ANOVA), and treatment effect sizes will be calculated. The durability of treatment gains will be examined by conducting repeated measures MANOVA followed by ANOVAs on scores obtained by treatment groups at times 0,1 and 2. A priori sample size calculations (outlined below) are computed for t-tests, with post hoc power calculations of observed power proposed for (M)ANOVA.

Considering clinical improvement rates, internationally used clinical cut-off scores for the HADS and PHQ-9/ GAD-7 will be employed. These cut-off scores will be used to determine clinical improvement. Cases who score 
above these cut-offs at time 0 and below them at time 1 or 2 will be classified as clinically improved at time 1 or 2. The statistical significance of intergroup differences in clinical improvements rates will be assessed with $\chi^{2}$ tests. To compare rates of reliable improvement in treatment and control groups on anxiety and depression scales, cases will be classified as reliably improved if they achieve a score $>1.96$ on the reliable change index (RCI) at time 1 or $2 .^{57}$ Cases will be classified as in remission at time 1 or 2 if they obtain scores below the clinical cut-off score at time 1 or 2 and achieve a score $>1.96$ on the RCI at time 1 or 2 . In relation to mediating factors, we will consider the cognitive and behavioural profile of the patient with ALS whom the caregiver provides care for. This will be done by calculating the relative risk and OR of a patient's cognitive and behavioural status defined categorically as the relative risk factor.

\section{Sample size}

To determine an appropriate sample size for the trial, a power analysis was conducted with $\mathrm{G}^{*}$ Power $3.1 .^{58} \mathrm{~A}$ power analysis indicated that in order for one-tailed statistical tests with $p$ values of 0.05 and power values of 0.90 to detect small-to-moderate differences $(\mathrm{d}=0.42)$ between three groups, a sample size of 75 study-completers (25 cases per cell) will be required, as this is the minimal test statistic and group size required. The effect size and dropout rate in this power analysis are based on results of relevant meta-analyses of psychological intervention groups. The estimated dropout rate of $50 \%$ is based on a meta-analysis by Fernandez et $a l^{59}$ who found average dropout rates of $39 \%-58 \%$. These rates include dropout before and during treatment. Therefore, participants will be recruited until the expected outcome of $n=25$ per cell is met. A post hoc power analysis will be completed to consider the proposed (M)ANOVA.

The estimated effect size of approximately 0.4 is based on results of meta-analyses. ${ }^{60-62}$ In a meta-analysis of 13 studies, Ebert $e t a b^{60}$ found that the average depression and anxiety symptom effect size for CBT compared with predominantly waiting list control groups was 0.72 . Reynolds $e t a l^{61}$ conducted a meta-analysis of 55 studies of CBT for anxiety. They found an average anxiety symptom effect size of 0.76 for CBT, delivered in both individual and group format, compared with waiting list control groups. However, they also found average effect sizes of 0.35 for studies evaluating brief CBT programmes of five to eight sessions; 0.57 for studies evaluating group rather than individual CBT programmes of any duration and 0.35 where CBT was compared with another active treatment. Cuijpers et at ${ }^{62}$ conducted a meta-analysis which found an effect size of 0.35 for depressive symptom based on comparisons with waiting list controls or treatment as usual. Based on these data and information, it is expected that this study will be adequately powered to detect significance with a cohort of 25 per arm of the study.

\section{Informed consent process}

The participant information sheet will explain the evidence-based approach of the interventions for mild-tomoderate anxiety and depression, as well as the nature of the treatment as usual group. Each programme will be briefly described. It will be explained that, in order to evaluate which programme works better, caregivers will be randomly assigned. It will also be explained that participants may withdraw from treatment at any time, and that opting not to participate in the research programme will not affect receipt of services, for them or the person with ALS. Participants will be provided with contact details of members of the research team should they wish to ask any further questions, and participants will be given time to read the information at their leisure. Participants are then asked on the day of consenting if they have further queries or questions. For the purpose of the CONSORT flow diagram, and in the interest of improving future research, participants who discontinue their engagement with the research will be provided the opportunity to express the reason for this. Informed consent of participants will be obtained (model consent form included as online supplement).

\section{DISCUSSION}

The results of this study will determine whether these group-based interventions have a role in mediating self-reported anxiety, depression and distress while also reducing subjective burden and improving QoL in caregivers of patients with ALS. Furthermore, findings will provide evidence to inform guidelines for future interventions tailored for ALS caregivers.

Recent longitudinal findings suggest that in ALS patient-caregiver dyads caregivers' experience of burden is associated with a higher baseline of anxiety and depression, which leads to a reduced QoL as the disease progresses. Further research within the field suggests that the presence of anxiety and low mood in caregivers at their initial clinic visit is predictive of developing caregiver burden as ALS progresses. ${ }^{32}$ As clinically elevated levels of burden have been reported in approximately $50 \%$ of caregivers, ${ }^{12}$ it is timely to investigate whether a reduction in anxiety and/or depression in caregivers can be achieved in a cost-effective group-based format. While there are multiple genetic, neurobiological, psychological and social factors that precipitate, predispose and perpetuate depression and anxiety, ${ }^{35-37}$ there is also a growing body of evidence from controlled trials, meta-analyses and systematic reviews that group-based interventions can be effective in treating mild-to-moderate anxiety and depression. ${ }^{38}$

The strengths of this study are its preventative, randomised trial design, thus the results are more likely to be generalisable to other ALS centres and ALS caregiver populations. Another strength is that this study will examine multiple important outcomes, while also considering factors such as the cognitive and behavioural 
phenotype of the person with ALS. Strengths also include the manualised approach to psychological intervention. Furthermore, we are somewhat uniquely placed internationally to assess the impact of interventions in caregivers of patients, as systemic support is not routinely available and thus the TAU group are unlikely to be exposed to any form of intervention.

A logistical issue for this study is the progressive and rapid nature of ALS, and the heterogenous disease profile, which may lead to an increase in caregiver dropout. As such, our power calculations are based on study completers, rather than on enrolment quota.

\section{CONCLUSION}

International awareness of burden and psychological distress in caregivers of patients with ALS has outpaced the available evidence on caregiver-specific treatments for this cohort. If found to be effective and efficacious, group-based interventions for caregivers of people with ALS could provide a routinely available intervention that could optimise caregivers' well-being, with an enrolment pathway possible at diagnosis to significantly reduce the burden of caregiving. This study will also enable a future evaluation of other treatment modalities, although as it stands, whether group-based interventions are useful for this cohort remains essentially untested.

Contributors TB, JWO'R, $\mathrm{OH}$ and NP were involved in conception and trial design. TB, JWO'R, SM, MG, MH, OH and NP were involved in drafting of the article and critical revision of the article for important intellectual content. TB, JWO'R, SM, MG, $\mathrm{MH}, \mathrm{OH}$ and NP were involved in final approval of the article.

Funding This research has been awarded funding by the ALS Association (ALSA; www.alsa.org).

Disclaimer The study funders had no role in conception, trial design or development of this protocol.

Competing interests None declared.

Patient consent for publication Not required.

Ethics approval This study has ethical approval from Beaumont Hospital's Research Ethics Committee (REC REF: 18/33).

Provenance and peer review Not commissioned; externally peer reviewed.

Open access This is an open access article distributed in accordance with the Creative Commons Attribution Non Commercial (CC BY-NC 4.0) license, which permits others to distribute, remix, adapt, build upon this work non-commercially, and license their derivative works on different terms, provided the original work is properly cited, appropriate credit is given, any changes made indicated, and the use is non-commercial. See: http://creativecommons.org/licenses/by-nc/4.0/.

\section{REFERENCES}

1. Burke T, Pinto-Grau M, Lonergan $\mathrm{K}$, et al. A cross-sectional population-based investigation into behavioral change in amyotrophic lateral sclerosis: subphenotypes, staging, cognitive predictors, and survival. Ann Clin Trans/ Neurol 2017;4:305-17.

2. Elamin M, Phukan J, Bede P, et al. Executive dysfunction is a negative prognostic indicator in patients with ALS without dementia. Neurology 2011;76:1263-9.

3. Pinto-Grau M, Costello E, O'Connor S, et al. Assessing behavioural changes in ALS: cross-validation of ALS-specific measures. J Neurol 2017;264:1397-401.

4. Radakovic R, Stephenson L, Newton J, et al. Multidimensional apathy and executive dysfunction in amyotrophic lateral sclerosis. Cortex 2017;94:142-51.
5. Abdulla S, Vielhaber S, Machts $\mathrm{J}$, et al. Information needs and information-seeking preferences of ALS patients and their carers. Amyotroph Lateral Scler Front Degener 2014;15:505-12.

6. Goldstein LH, Adamson M, Jeffrey L, et al. The psychological impact of MND on patients and carers. J Neurol Sci 1998;160:S114-S121.

7. Pagnini F, Rossi G, Lunetta C, et al. Burden, depression, and anxiety in caregivers of people with amyotrophic lateral sclerosis. Psychol Heal Med 2010;15:685-93.

8. Burke T, Elamin M, Galvin M, et al. Caregiver burden in amyotrophic lateral sclerosis: a cross-sectional investigation of predictors. $J$ Neurol 2015;262:1526-32.

9. Chiò A, Gauthier A, Calvo A, et al. Caregiver burden and patients' perception of being a burden in ALS. Neurology 2005;64:1780-2.

10. Gauthier A, Vignola A, Calvo A, et al. A longitudinal study on quality of life and depression in ALS patient-caregiver couples. Neurology 2007;68:923-6.

11. Burke T, Galvin M, Pinto-Grau M, et al. Caregivers of patients with amyotrophic lateral sclerosis: investigating quality of life, caregiver burden, service engagement, and patient survival. J Neurol 2017; 264:898-904.

12. Lillo $P$, Mioshi $E$, Hodges JR. Caregiver burden in amyotrophic lateral sclerosis is more dependent on patients' behavioral changes than physical disability: a comparative study. BMC Neurol 2012;12:156.

13. Tremolizzo L, Pellegrini A, Susani E, et al. Behavioural but not cognitive impairment is a determinant of caregiver burden in amyotrophic lateral sclerosis. Eur Neurol 2016;75:191-4.

14. Bruletti G, Comini L, Scalvini S, et al. A two-year longitudinal study on strain and needs in caregivers of advanced ALS patients. Amyotroph Lateral Scler Front Degener 2015;16:187-95.

15. Chiò Aet al. A cross sectional study on determinants of quality of life in ALS. J Neurol Neurosurg Psychiatry 2004;75:1597-601.

16. Mioshi E, Caga J, Lillo P, et al. Neuropsychiatric changes precede classic motor symptoms in ALS and do not affect survival. Neurology 2014;82:149-55.

17. Siciliano M, Santangelo G, Trojsi F, et al. Coping strategies and psychological distress in caregivers of patients with amyotrophic lateral sclerosis(ALS). Amyotroph Lateral Scler Front Degener 2017;18:367-77.

18. Rabkin JG, Wagner GJ, Del Bene M. Resilience and distress among amyotrophic lateral sclerosis patients and caregivers. Psychosom Med 2000;62:271-9.

19. Burke T, Galvin M, Maguire S. The impact of cognitive and behavioural change on quality of life of caregivers and patients with ALS and other neurological conditions. In: Amyotrophic lateral sclerosis: understanding and optimizing quality of life and psychological well-being. Oxford: Oxford University Press, 2018: 151.

20. Martín-Carrasco M, Martín MF, Valero CP, et al. Effectiveness of a psychoeducational intervention program in the reduction of caregiver burden in Alzheimer's disease patients' caregivers. Int J Geriatr Psychiatry 2009;24:489-99.

21. Acton GJ, Kang J. Interventions to reduce the burden of caregiving for an adult with dementia: a meta-analysis. Res Nurs Health 2001;24:349-60.

22. Choi A-N, Lee MS, Cheong K-J, et al. Effects of group music intervention on behavioral and psychological symptoms in patients with dementia: a pilot-controlled trial. Int $J$ Neurosci 2009;119:471-81.

23. Mittelman MSet al. A family intervention to delay nursing home placement of patients with Alzheimer disease. JAMA 1996;276.

24. Larouche E, Hudon C, Goulet S. Potential benefits of mindfulnessbased interventions in mild cognitive impairment and Alzheimer's disease: an interdisciplinary perspective. Behav Brain Res 2015;276:199-212

25. Pagnini F, Phillips D, Bosma CM, et al. Mindfulness as a protective factor for the burden of caregivers of amyotrophic lateral sclerosis patients. J Clin Psychol 2016;72:101-11.

26. Murphy V, Felgoise SH, Walsh SM, et al. Problem solving skills predict quality of life and psychological morbidity in ALS caregivers. Amyotrophic Lateral Sclerosis 2009;10:147-53.

27. Ng L, Amatya B, Khan F. Outcomes of a peer support program in multiple sclerosis in an Australian community cohort: a prospective study. J Neurodegener Dis 2013;2013:1-7.

28. Dowrick C, Dunn G, Ayuso-Mateos JL, et al. Problem solving treatment and group psychoeducation for depression: multicentre randomised controlled trial. outcomes of depression international network(Odin)group. BMJ 2000;321:1450-4.

29. Zoccolella $S$, Beghi E, Palagano G, et al. Als multidisciplinary clinic and survival. J Neurol 2007;254:1107-12.

30. Abrahams S, Newton J, Niven E, et al. Screening for cognition and behaviour changes in ALS. Amyotroph Lateral Scler Front Degener 2014;15:9-14. 
31. Higginson IJ, Carr AJ. Measuring quality of life: using quality of life measures in the clinical setting. BMJ 2001;322:1297-300.

32. Burke T, Hardiman O, Pinto-Grau M, et al. Longitudinal predictors of caregiver burden in amyotrophic lateral sclerosis: a population-based cohort of patient-caregiver dyads. J Neurol 2018;265:793-808.

33. de Wit J, Bakker LA, van Groenestijn AC, et al. Caregiver burden in amyotrophic lateral sclerosis: a systematic review. Palliat Med 2018;32:231-45.

34. Chen D, Guo X, Zheng Z, et al. Depression and anxiety in amyotrophic lateral sclerosis: correlations between the distress of patients and caregivers. Muscle Nerve 2015;51:353-7.

35. Essau CA, Ollendick TH. The Wiley-Blackwell Handbook of the treatment of childhood and adolescent anxiety, 2012.

36. Higa-McMillan CK, Francis SE, Rith-Najarian L, et al. Evidence base update: 50 years of research on treatment for child and adolescent anxiety. J Clin Child Adolesc Psychol 2016;45:91-113.

37. Maalouf FT, Brent DA. Depressive disorders in children. In: Encyclopedia of Psychopharmacology, 2015.

38. Kendall PC, Peterman JS. Cbt for adolescents with anxiety: mature yet still developing. Am J Psychiatry 2015;172:519-30.

39. van Groenestijn AC, Schröder CD, Visser-Meily JMA, et al. Cognitive behavioural therapy and quality of life in psychologically distressed patients with amyotrophic lateral sclerosis and their caregivers: results of a prematurely stopped randomized controlled trial. Amyotroph Lateral Scler Front Degener 2015;16:309-15.

40. Caga J, Hsieh S, Lillo P, et al. The impact of cognitive and behavioral symptoms on ALS patients and their caregivers. Front Neurol 2019;10.

41. Ho FY-Y, Yeung W-F, Ng TH-Y, et al. The efficacy and costeffectiveness of stepped care prevention and treatment for depressive and/or anxiety disorders: a systematic review and metaanalysis. Sci Rep 2016;6:29281

42. van Straten A, Hill J, Richards DA, et al. Stepped care treatment delivery for depression: a systematic review and meta-analysis. Psychol Med 2015;45:231-46.

43. Schulz KF, Altaian DG. Statement: updated guidelines for reporting parallel group randomized trials. Japanese Pharmacol. Ther 2010;340.

44. Lorig K, Thompson-Gallagher D, Traylor L, et al. Building better caregivers: a pilot online support workshop for family caregivers of cognitively impaired adults. J Appl Gerontol 2012.

45. Santorelli S, Meleo-Meyer F, Koerbel L. Mindfulness-based stress reduction (MBSR): authorized curriculum guide, 2017.

46. McDonnell E, Forry M, O'Raghallaigh JW, et al. Pilot study of a multitiered psychosocial support framework for inflammatory bowel disease patients. Gastrointest Nurs 2014;12:42-9.

47. Kroenke K, Spitzer RL, Williams JB. The PHQ-9: validity of a brief depression severity measure. J Gen Intern Med 2001;16:606-13.

48. Spitzer RL, Kroenke K, Williams JBW, et al. A brief measure for assessing generalized anxiety disorder: the GAD-7. Arch Intern Med 2006;166:1092.
49. Zigmond AS, Snaith RP. The hospital anxiety and depression scale. Acta Psychiatr Scand 1983;67:361-70.

50. Zarit SH, Reever KE, Bach-Peterson J. Relatives of the impaired elderly: correlates of feelings of burden. Gerontologist 1980;20:649-55.

51. Cohen SR, Mount BM, Strobel MG, et al. The McGill quality of life questionnaire: a measure of quality of life appropriate for people with advanced disease. A preliminary study of validity and acceptability. Palliat Med 1995;9:207-19.

52. Cedarbaum JM, Stambler N, Malta E, et al. The ALSFRS-R: a revised ALS functional rating scale that incorporates assessments of respiratory function. BDNF ALS Study Group(phase III). J Neurol Sci 1999;169:13-21.

53. Niven E, Newton J, Foley J, et al. Validation of the Edinburgh cognitive and behavioural amyotrophic lateral sclerosis screen (ECAS): a cognitive tool for motor disorders. Amyotroph Lateral Scler Front Degener 2015;16:172-9.

54. Elamin M, Pinto-Grau M, Burke T, et al. Identifying behavioural changes in ALS: validation of the Beaumont behavioural inventory(BBI). Amyotrophic Lateral Sclerosis and Frontotemporal Degeneration 2017;18:68-73.

55. Pinto-Grau M, Burke T, Lonergan $\mathrm{K}$, et al. Screening for cognitive dysfunction in ALS: validation of the Edinburgh cognitive and behavioural ALS screen (ECAS)using age and education adjusted normative data. Amyotroph Lateral Scler Front Degener 2017;18:99-106.10.1080/21678421.2016.1249887

56. Bryan CJ, Gartner AM, Wertenberger E, et al. Defining treatment completion according to patient competency: a case example using brief cognitive behavioral therapy(BCBT)for suicidal patients. Prof Psychol Res Pract 2012.

57. Jacobson NS, Truax P. Clinical significance: a statistical approach to defining meaningful change in psychotherapy research. $J$ Consult Clin Psychol 1991;59:12-19.

58. Faul F, Erdfelder E, Lang A-G, et al. G*Power 3: a flexible statistical power analysis program for the social, behavioral, and biomedical sciences. Behav Res Methods 2007;39:175-91.

59. Fernandez E, Salem D, Swift JK, et al. Meta-Analysis of dropout from cognitive behavioral therapy: magnitude, timing, and moderators. $J$ Consult Clin Psychol 2015;83:1108-22.

60. Ebert DD, Zarski A-C, Christensen $\mathrm{H}$, et al. Internet and computerbased cognitive behavioral therapy for anxiety and depression in youth: a meta-analysis of randomized controlled outcome trials. PLoS One 2015;10:e0119895.

61. Reynolds S, Wilson C, Austin J, et al. Effects of psychotherapy for anxiety in children and adolescents: a meta-analytic review. Clin Psychol Rev 2012;32:251-62.

62. Cuijpers P, Marks IM, van Straten A, et al. Computer-Aided psychotherapy for anxiety disorders: a meta-analytic review. Cogn Behav Ther 2009;38:66-82. 